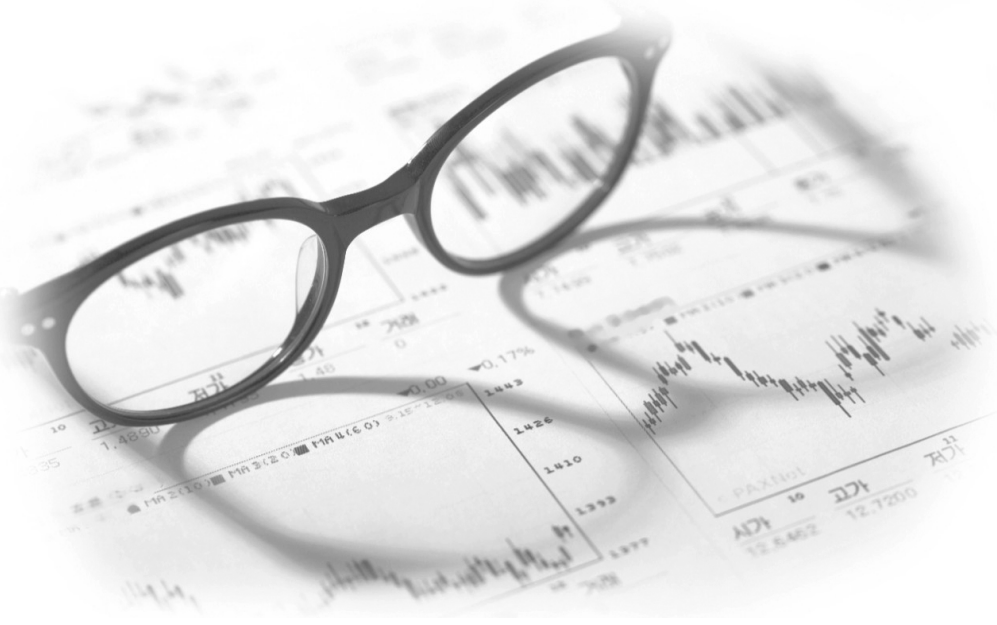

BLOOD RESEARCH

Volume 51 • Number 4 - December 2016

https://doi.org/10.5045/br.2016.51.4.220

Perspective

\section{Salvage treatment of relapsed/refractory LCH}

\author{
Jong Jin Seo, M.D., Ph.D. \\ Department of Pediatrics, Division of Hematology-Oncology, \\ University of Ulsan College of Medicine \& Asan Medical \\ Center, Seoul, Korea
}

Received on December 12, 2016; Accepted on December 19, 2016

\section{Introduction}

The Langerhan's cell histiocytosis (LCH) is a rare hematologic disorder of all age group, and the classification of LCH as a neoplasm of myeloid origin was introduced with the current observation of recurrent $B R A F^{V 600 E}$ mutation in $\mathrm{LCH}$ patients. LCH shows broad spectrum of clinical manifestations from single system involvement to wide spread multi-system (MS) disease, and this clinical variety is assumed to be determined by the differentiation stage of myeloid dendritic cells in which the mitogen-activated protein kinase (MAPK) pathway is aberrantly activated. The lesions of LCH typically show clonal proliferation of $\mathrm{CD}^{+} \mathrm{a}^{+} / \mathrm{CD} 207^{+}$cells with significant infiltration of inflammatory cells, and the documentation of this $\mathrm{CD}^{+} \mathrm{a}^{+}$or $\mathrm{CD}_{20} \mathrm{7}^{+}$ cell in biopsy specimen is required for the diagnosis of LCH.

The survival of relapsed/refractory $(\mathrm{R} / \mathrm{R})$ high risk LCH patients was significantly improved with intensification of therapy, and the disease reactivation rate was somewhat reduced overtime due to prolongation of therapy during last two decades. Despite this improvements in survival and reactivation rate, the risk of permanent consequences of LCH was only marginally reduced [1].

Although most of the relapsed patients without risk organ involvement (RO-) can be salvaged successfully with less intensive chemotherapies, they are more likely to develop permanent consequences. The poor survival rate of MS LCH patients with $\mathrm{RO}$ involvement $(\mathrm{RO}+)$ who are refractory to or relapsing after standard chemotherapy was much improved with the introduction of salvage chemotherapy using $2 \mathrm{CdA}$ and Ara-C [2], but still there are patients not responding and dying with $\mathrm{R} / \mathrm{R}$ disease.

Therefore, the intensity and duration of second line treatment for these R/R MS LCH patients should be decided according to the status of RO involvement. LCH-IV protocols of the Histiocyte Society also employ the strategies of salvage therapy for patients with $\mathrm{R} / \mathrm{R} \mathrm{LCH}$ according to the status of $\mathrm{RO}$ involvement. The second line treatment for $\mathrm{R} / \mathrm{R} \mathrm{RO}-\mathrm{LCH}$ patient needs to achieve disease resolution, prevent further reactivations and permanent consequences. Moreover, the goal of second line treatment for $\mathrm{R} / \mathrm{R} \mathrm{RO}+$ $\mathrm{LCH}$ patient needs to include further reduction of mortality due to disease progression as well.

\section{Salvage treatment for relapsed/refractory low risk $\mathrm{LCH}$}

Various medications such as oral 6-MP and methotrexate, indomethacin, bisphosphonates, BRAF inhibitors, lower dose $2 \mathrm{CdA}$, Ara-C, and clofarabine were reported to be effective for patients with $\mathrm{R} / \mathrm{R} \mathrm{RO}-\mathrm{LCH}$, but the most optimal treatment for R/R RO- LCH patient is not determined yet [3-5].

Current LCH-IV protocol of the Histiocyte Society adopts a uniform second line treatment of intensive 24-week initial chemotherapy using prednisone, Ara-C and vincristine for all R/R RO- low risk LCH patients (Stratum II). Those patients who have responded, i.e. non-active disease (NAD) and active disease better ( $\mathrm{AD}$ better), after this initial therapy are randomly assigned to either of the two continuation 
arms, indomethacin vs. 6-MP/Methotrexate, and treated to a total treatment duration of 24 months including initial therapy. The primary aim of this stratum is a reduction of the reactivation rate, and the 2 arms of continuation therapy aims to investigate the efficacy of indomethacin, an acceptable non-toxic alternative drug, in preventing further reactivations against the standard 6-MP/methotrexate combination.

\section{Salvage treatment for relapsed/refractory high risk $\mathrm{LCH}$}

There had been several reports showing the effectiveness of $2 \mathrm{CdA} /$ Ara-C combination as second line treatment for $\mathrm{R} / \mathrm{R}$ MS RO+ high risk LCH patients [2].

Two promising therapeutic strategies for the treatment for $\mathrm{R} / \mathrm{R} \mathrm{RO}+$ patients are being evaluated through ongoing prospective LCH-IV study. One is the combination chemotherapy with 2-CdA and Ara-C (Stratum III), and the other is allogeneic hematopoietic stem cell transplantation (HSCT) (Stratum IV) [6].

As the 2-CdA/Ara-C chemotherapy frequently develop WHO grade 4 hematologic toxicity and severe infection [2], the usage of this regimen should be restrictive to patients showing "AD intermediate or $\mathrm{AD}$ worse" in $\mathrm{RO}$ after week 6 or week 12 of standard chemotherapy with vinblastine and prednisone. Those MS RO+ patients who are regarded as "AD better" without achieving NAD state after 12 weeks' standard therapy need to be treated with less toxic vincristine/Ara-C/prednisone chemotherapy of Stratum II. For further therapy, patients need to be stratified according to the treatment response after 2 courses of $2-\mathrm{CdA} /$ Ara- $\mathrm{C}$ regimen. For those patients with $\mathrm{AD}$ worse after 2 courses compared to pre-salvage assessment, reduced intensity conditioning (RIC) HSCT according to Stratum IV is recommended to improve the survival. RIC-HSCT was reported to be a preferable procedure with low transplantrelated morbidity and mortality $[4,5]$, but recent study revealed no significant difference in transplant-related mortality by conditioning regimen intensity [6]. The relapse rate was higher after RIC compared to myeloablative conditioning (MAC) regimens, although most patients relapsing after RIC transplantation could be salvaged with further chemotherapy [6].

It is not yet clear whether the 2-CdA/Ara-C salvage therapy (Stratum III) or RIC-HSCT (Stratum IV) is the superior approach for refractory $\mathrm{RO}+$ high risk patients, and therefore either option can be selected depending on physician's choice and donor availability as part of the LCH-IV Study.

There were case reports showing the efficacy of clofarabine monotherapy [3] and steroid pulse with lenalidomide [7] as a successful salvage therapy in LCH patients who failed to achieve a durable response to 2-CdA/Ara-C.

\section{Perspectives}

The ongoing prospective LCH-IV studies for the R/R LCH will give important information on the better regimen to further reduce the reactivation rate and permanent sequelae for $\mathrm{R} / \mathrm{R} \mathrm{RO}-$ patients and the mortality for $\mathrm{R} / \mathrm{R} \mathrm{RO}+$ patients.

The controversies in the conditioning regimen of HSCT for LCH patients still remain to be clarified with further studies comparing RIC and MAC regimen in larger cohort of LCH patients.

It seems to be unlikely that significant survival improvements can be achieved with current cytotoxic chemotherapy regimen alone due to the high toxicity associated with it, which necessitate a need for noble therapeutic approaches.

Targeted therapies using inhibitors for the recurrent mutations in MAPK pathway which underlie the pathogenesis of LCH, such as BRAF or MEK inhibitors, were reported to be uniformly effective for patients with these mutations. However further investigation on the efficacy and long-term safety in childhood LCH patients is needed before their clinical use $[4,5,8-10]$.

Recent advances in the knowledge on the biology of LCH may facilitate a change in the current therapeutic strategy employing the risk stratification based on the disease extent and site. The biology studies of current prospective clinical trial are expected to reveal important information on putative biomarkers of LCH which can be incorporated in the strategy of risk stratification for personalized treatment aiming further improvement in the survival of patients with $\mathrm{R} / \mathrm{R} \mathrm{RO}+\mathrm{LCH}$.

\section{Acknowledgements}

This study was supported by a grant (2016-E63002-00) from Korea Centers for Disease Control and Prevention.

\section{Author's Disclosure of Potential Conflict of Interest}

The author has no potential conflict of interest relevant to this article.

\section{REFERENCES}

1. Rigaud C, Barkaoui MA, Thomas C, et al. Langerhans cell histiocytosis: therapeutic strategy and outcome in a 30-year nationwide cohort of 1478 patients under 18 years of age. Br J Haematol 2016;174:887-98.

2. Donadieu J, Bernard F, van Noesel M, et al. Cladribine and cytarabine in refractory multisystem Langerhans cell histiocytosis: results of an international phase 2 study. Blood 2015;126:1415-23.

3. Simko SJ, Tran HD, Jones J, et al. Clofarabine salvage therapy in refractory multifocal histiocytic disorders, including Langerhans cell histiocytosis, juvenile xanthogranuloma and Rosai-Dorfman disease. Pediatr Blood Cancer 2014;61:479-87.

4. Monsereenusorn C, Rodriguez-Galindo C. Clinical Characteris- 
tics and treatment of Langerhans cell histiocytosis. Hematol Oncol Clin North Am 2015;29:853-73.

5. Allen CE, Ladisch S, McClain KL. How I treat Langerhans cell histiocytosis. Blood 2015;126:26-35.

6. Veys PA, Nanduri V, Baker KS, et al. Haematopoietic stem cell transplantation for refractory Langerhans cell histiocytosis: outcome by intensity of conditioning. Br J Haematol 2015;169: 711-8.

7. Uppuluri R, Ramachandrakurup S, Subburaj D, Bakane A, Raj R. Excellent remission rates with limited toxicity in relapsed/ refractory Langerhans cell histiocytosis with pulse dexamethasone and lenalidomide in children. Pediatr Blood Cancer
2017;64:110-2.

8. Haroche J, Cohen-Aubart F, Emile JF, et al. Dramatic efficacy of vemurafenib in both multisystemic and refractory ErdheimChester disease and Langerhans cell histiocytosis harboring the BRAF V600E mutation. Blood 2013;121:1495-500.

9. Aricò M. Langerhans cell histiocytosis in children: from the bench to bedside for an updated therapy. Br J Haematol 2016;173: 663-70.

10. Héritier S, Emile JF, Barkaoui MA, et al. BRAF mutation correlates with high-risk Langerhans cell histiocytosis and increased resistance to first-line therapy. J Clin Oncol 2016;34:3023-30. 